Proyecciones

Vol. 20, No 1, pp. 01-17, May 2001.

Universidad Católica del Norte

Antofagasta - Chile

\title{
CONJUGACIES CLASSES OF SOME NUMERICAL METHODS*
}

\author{
SERGIO PLAZA \\ Universidad de Santiago, Santiago-Chile
}

\begin{abstract}
We study the dynamics of some numerical root finding methods such as the Newton, Halley, König and Schröder methods for three and four degree complex polynomials.
\end{abstract}

*Part of this work was supported by FONDECYT Grants \#1970720 and \#1961212, and DICYT Grant \#9733 P.S. 


\section{Introduction}

Let $p(z)$ be a complex polynomial. The Newton iteration function associated to $p(z)$ is

$$
N_{p}(z)=z-\frac{p(z)}{p^{\prime}(z)}
$$

The function $N_{p}$ defines a rational map on the Riemann sphere $\overline{\mathbf{C}}=\mathbf{C} \cup\{\infty\}$ and, thus, defines a discrete dynamical system $z_{n+1}=$ $N_{p}\left(z_{n}\right)$. If the seed $z_{0}$ is chosen "near" a root $\alpha$ of $p(z)$, then the sequence $\left(z_{n}\right)_{n \in \mathbf{N}}$ converges quadratically to the root $\alpha$, since $\alpha$ is a superattracting fixed point of $N_{p}$. This fact makes the Newton method one of the most used for approximating the roots of a polynomial. In [19] Haeseler and Peitgen discuss the basin of attraction of the roots and give an overview of complex dynamics of rational maps on the Riemann sphere. For an extensive and comprehensive review of the theory of iteration of rational maps, see that given by Blanchard in [7], and that by Blanchard and Chiu in [8], and for a treatment of the theory see Milnor [18], and Emerenko and Lyubich [16]. Concerning a history of complex dynamics, the reader can consult, e.g., Alexander [1] and the reference therein.

For the study of other iteration functions used for finding a root of a polynomial, see [21] and [22] in which the dynamics of the Schröder and König iteration functions is studied. The parameter space associated to Schröder and König iteration functions of the one-parameter family of cubic polynomials $p_{A}(z)=z^{3}+(A-1) z-A$, is studied in [22]. In [11] This kind of study was initiated by Curry, Garnett and Sullivan for the Newton iteration function associated to the one-parameter family $p_{A}(z)$ of above. Here parameter regions where extraneous attractive periodic cycles exist are described. This feature is also observed for the Schröder iteration function associated to $p_{A}(z)$ (see [21]) as well as for the König iteration function associated to $p_{A}(z)$ (see [22]). A more recent study on this subject is given in [12]. Another well known iteration function for finding roots of a polynomial is the Halley iteration function; for a study of this iteration function see [6] and [5] and the references therein.

In Section 3 the study of the dynamics of Newton iteration function 
associated to a degree three generic polynomial is reduced (by conjugacy) to the study of the dynamics of the Newton iteration function of $p_{\rho}(z)=z(z-\rho)(z-1)$ (equivalently, of $p_{A}(z)=z^{3}+(A-1) z-A$ ), for the corresponding parameter value. The same is done for the Halley iteration function as well as for Schröder and König iteration functions in sections 4,5 , and 6 , respectively,.

\section{Preliminaries}

We recall the following definition.

Definition 1. Let $f$ and $g$ be two maps from the Riemann sphere into itself. An analytic conjugacy between $f$ and $g$ is an analytical diffeomorphism $h$ from the Riemann sphere into itself such that $h \circ$ $f=g \circ h$ (conjugacy equation).

Remark. A conjugacy $h$ between two maps $f$ and $g$ preserves the basic feature of the dynamics; for example, $h$ sends periodic points of $f$ into periodic points of $g$ and so on.

Now we recall the concept of order of convergence of an analytical function in a neighborhood of an attracting fixed point. Let $F$ : $\mathbf{C} \rightarrow \mathbf{C}$ be an analytic function, and let $z_{0} \in \mathbf{C}$. We define the sequence of iterates of $z_{0}$ by $F$ as $\left(z_{n}\right)_{n \in \mathbf{N}}$ where $z_{n+1}=F\left(z_{n}\right)$, $n=0,1,2, \ldots$. Assume that $\lim _{n \rightarrow \infty} z_{n}=\tilde{z}$. The error associated to the $n$-th iterate is given by $e_{n}=z_{n}-\tilde{z}$. If $m$ is the first positive integer such that $F^{(m)}(\tilde{z}) \neq 0$ we have

$e_{n+1}=z_{n}-\tilde{z}=F\left(e_{n}+\tilde{z}\right)-F(\tilde{z})=\frac{1}{m !} F^{(m)}(\tilde{z}) e_{n}^{m}+O\left(e_{n}^{m+1}\right), \quad n \rightarrow \infty$.

In this case we say that $F$ is an order $m$ iteration function. For example, generically, the Newton iteration function has order 2 .

\section{The Newton Iteration Function}

We have the following useful and well known result ([13]). 
Theorem 1. (Reescaling) Let $T(z)=\alpha z+\beta, \alpha \neq 0$ be an affine map, and let $q(z)=p \circ T(z)$. Then

$$
T \circ N_{q} \circ T^{-1}=N_{p}
$$

that is, $T$ is a conjugacy between $N_{p}$ and $N_{q}$.

We restrict our attention to degree three and four generic polynomials. For non generic polynomials (that is, polynomials with multiple roots) the Newton iteration function associated to such polynomials does not have good properties. For example, in a neighborhood of a multiple root, the convergence of iterations to it is only linear. For a study of the Newton method for multiple roots see [14] and [15].

For the sake of completeness, we now give a result for the Newton iteration function for generic quadratic polynomials.

Proposition 1. Let $p(z)=a z^{2}+b z+c$, with $a \neq 0$, be a generic quadratic polynomial, and let $q(z)=z^{2}-A$ where $A=b^{2}-4 a c$. Then $N_{p}$ and $N_{q}$ are conjugated.

Remark The study of the dynamics of the Newton iteration function associated to a generic quadratic polynomial was done by A. Cayley in [9] and [10].

Let us consider generic cubic polynomials; that is, polynomials with their three roots distinct.

Theorem 2. Let $p(z)$ be a generic cubic polynomial, and let $a, b$ and $c$ be its roots. Assume that they are ordered as follows: $0 \leq$ $|a| \leq|b| \leq|c|$. Let $T(z)=(c-a) z+a$. Then $q_{\lambda, \rho}(z)=p \circ T(z)=$ $\lambda^{3} z(z-\rho)(z-1)$, where $\lambda=c-a$ and $\rho=\frac{b-a}{c-a}$ (note that $|\rho|<1$ ), and $T$ is a conjugacy between $N_{p}$ and $N_{q}\left(q(z)=q_{\lambda, \rho}(z)\right)$; that is, $T^{-1} \circ N_{q} \circ T=N_{p}$. Furthermore, if $\tilde{q}_{\rho}(z)=z(z-1)(z-\rho)$, then $N_{p}$ is conjugated with $N_{\tilde{q}}$.

Proof. The proof follows from Theorem 1 of above and a straightforward computation.

Note that, for three degree generic polynomials, the parameter $\lambda$ of above is not essential in the study of the dynamics of the Newton map for three degree generic polynomials. 
Now we show how our one-parameter family $\tilde{q}_{\rho}(z)=z(z-\rho)(z-1)$ may be reduced to the well known family $p_{A}(z)=z^{3}+(A-1) z-A$. For this, let $T(z)=\frac{2-\rho}{3} z+\frac{1+\rho}{3}$. Then

$$
\tilde{q}_{\rho} \circ T(z)=\left(\frac{2-\rho}{3}\right)^{3}\left(z-\frac{1+\rho}{\rho-2}\right)\left(z-\frac{1-2 \rho}{\rho-2}\right)(z-1) .
$$

Hence setting $\mu=\left(\frac{2-\rho}{3}\right)^{3}$ and $A=\frac{1-\rho-2 \rho^{2}}{(\rho-2)^{2}}$, the family $\tilde{q}_{\rho}(z)$ may be written as follows:

$$
\tilde{q}_{A, \mu}(z)=\tilde{q}_{\rho} \circ T(z)=\mu\left(z^{3}+(A-1) z-A\right),
$$

. Since $\tilde{q}_{A, \mu}(z)=\tilde{q}_{\rho} \circ T(z)$, we have $T \circ N_{\tilde{q}_{A, \mu}} \circ T^{-1}=N_{\tilde{q}_{\rho}}$ by the Scaling Theorem. On the other hand, considering the family $p_{A}(z)=$ $z^{3}+(A-1) z-A$ we have that $N_{p_{A}}(z)=N_{q_{A, \mu}}$. Therefore, we have reduced the study of the dynamics of $N_{\tilde{q}_{\rho}}$ to the study of the dynamics of $N_{p_{A}}$, which is simple. A study of the $A$-parameter space was done in [11].

Let us consider generic four degree polynomials; that is, polynomials with their four roots distinct.

Theorem 3. Let $p(z)=(z-a)(z-b)(z-c)(z-d)$ be a four degree generic polynomial. Assume that the roots of $p(z)$ are distinct and ordered as follows: $0 \leq|a| \leq|b| \leq|c| \leq|d|$. Let $T(z)=(d-a) z+a$, and let $q(z)=p \circ T(z)$. Then $T \circ N_{q_{\lambda, \rho, \mu}} \circ T^{-1}=N_{p}$ (that is, the Newton iteration functions of $q_{\lambda, \rho, \mu}(z)$ and of $p(z)$ are conjugated), where $q_{\lambda, \rho, \mu}(z)=p(T(z))=\lambda z(z-\rho)(z-\mu)(z-1), \lambda=(d-a)^{4}$, $\rho=\frac{b-a}{d-a}$, and $\mu=\frac{c-a}{d-a}$. Furthermore, $N_{q_{\lambda, \rho, \mu}}$ is conjugated to $N_{q_{\rho, \mu}}$ where $q_{\rho, \mu}(z)=z(z-\rho)(z-\mu)(z-1)$.

Proof. The proof follows from Theorem 1 of above and a straightforward computation.

Hence to study the dynamics of the Newton iteration function of a four degree generic polynomial, it suffices to study the dynamics of the Newton iteration function of the corresponding polynomial $q_{\rho, \mu}(z)$. A study of the $(\rho, \mu)$-parameter space may be done. 


\section{The Halley Iteration Function}

The Halley iteration function of a map is next in the order of simplicity of the root finding methods after the Newton iteration function.

Let $p(z)$ be a complex polynomial. The Halley iteration function associated to $p(z)$ is

$$
H_{p}(z)=z-\frac{2 p(z) p^{\prime}(z)}{2\left(p^{\prime}(z)\right)^{2}-p(z) p^{\prime \prime}(z)} .
$$

Let $\alpha$ be a simple root of $p(z)$. Then we have:

(a) $H_{p}(\alpha)=\alpha$; that is, $\alpha$ is a fixed point of $H_{p}$. Furthermore, $H_{p}^{\prime}(\alpha)=H_{p}^{\prime \prime}(\alpha)=0$ and $H_{p}^{\prime \prime \prime}(\alpha) \neq 0$, hence $H_{p}$ is an iteration function of order three in a neighborhood of $\alpha$;

(b) $H_{p}^{\prime \prime \prime}(\alpha)=-\left(\frac{p^{\prime \prime \prime}(\alpha)}{p^{\prime}(\alpha)}-\frac{3}{2}\left(\frac{p^{\prime \prime}(z)}{p^{\prime}(z)}\right)^{2}\right)=-S(p)(\alpha)$, where $S(p)(z)$ is the Schwarzian derivative of $p(z)$.

For two degree generic quadratic polynomials we have the following result.

Proposition 2. Let $p(z)=a z^{2}+b z+c$, with $a \neq 0$, be a generic quadratic polynomial, and let $q(z)=z^{2}-A$ where $A=b^{2}-4 a c$. Then $H_{p}$ and $H_{q}$ are conjugated. Then

Proof. We have $H_{q}(z)=z-\frac{2 z(z-A)}{3 z^{2}+A}$. Now let $\tau(z)=2 a z+b$.

$$
\begin{aligned}
& \tau \circ H_{p} \circ \tau^{-1}(z)=\tau\left(H_{p}\left(\tau^{-1}(z)\right)\right) \\
& =2 a H_{p}\left(\tau^{-1}(z)\right)+b \\
& =2 a\left(\tau^{-1}(z)-\frac{2 p\left(\tau^{-1}(z)\right) p^{\prime \prime}\left(\tau^{-1}(z)\right)}{2\left(p^{\prime}\left(\tau^{-1}(z)\right)\right)^{2}-p\left(\tau^{-1}(z)\right) p^{\prime \prime}\left(\tau^{-1}(z)\right)}\right)+b \\
& =2 a\left(\frac{z-b}{2 a}\right)-2 a \frac{2 p\left(\tau^{-1}(z)\right) f^{\prime \prime}\left(\tau^{-1}(z)\right)}{2\left(p^{\prime}\left(\tau^{-1}(z)\right)\right)^{2}-p\left(\tau^{-1}(z)\right) p^{\prime \prime}\left(\tau^{-1}(z)\right)}+b \\
& =z-2 a \frac{2 p\left(\tau^{-1}(z)\right) p^{\prime}\left(\tau^{-1}(z)\right)}{2\left(p^{\prime}\left(\tau^{-1}(z)\right)\right)^{2}-p\left(\tau^{-1}(z)\right) p^{\prime \prime}\left(\tau^{-1}(z)\right)}
\end{aligned}
$$


On the other hand, we have $p\left(\tau^{-1}(z)\right)=\frac{1}{4 a}\left(z^{2}+4 a c-b^{2}\right)$, $p^{\prime}\left(\tau^{-1}(z)\right)=z$, and $p^{\prime \prime}\left(\tau^{-1}(z)\right)=2 a$. Substituting these equalities in the expression of $\tau \circ H_{p} \circ \tau^{-1}$ we obtain

$$
\begin{aligned}
\tau \circ H_{p} \circ \tau^{-1}(z) & =z-\frac{2 a \cdot 2 \cdot \frac{1}{4 a}\left(z^{2}+4 a c-b^{2}\right) z}{2 z^{2}-\frac{1}{4 a}\left(z^{2}+4 a c-b^{2}\right) 2 a} \\
& =z-\frac{\left(z^{2}+4 a c-b^{2}\right) z}{\frac{3 z^{2}-\left(4 a c-b^{2}\right)}{2}} \\
& =z-\frac{2 z\left(z^{2}-A\right)}{3 z^{2}+A}=H_{q}(z) ;
\end{aligned}
$$

that is, $\tau \circ H_{p} \circ \tau^{-1}=H_{q}$, which completes the proof.

Proposition 3. (Scaling theorem for the Halley iteration function) Let $p(z)$ be a complex polynomial, and let $T(z)=\alpha z+\beta, \alpha \neq 0$ be an affine map. If $q(z)=p \circ T(z)$, then $T \circ H_{q} \circ T^{-1}(z)=H_{p}(z)$; that is, $H_{p}$ and $H_{q}$ are conjugated by $T$.

Proof. We have

$$
H_{q}\left(T^{-1}(z)\right)=T^{-1}(z)-\frac{2 q\left(T^{-1}(z)\right) q^{\prime}\left(T^{-1}(z)\right)}{2\left(q^{\prime}\left(T^{-1}(z)\right)^{2}-q\left(T^{-1}(z)\right) q^{\prime \prime}\left(T^{-1}(z)\right)\right.} .
$$

On the other hand, $q \circ T^{-1}(z)=p(z)$, thus $\left(q \circ T^{-1}\right)^{\prime}(z)=$ $\frac{1}{\alpha} q^{\prime}\left(T^{-1}(z)\right.$. Hence it follows that $q^{\prime}\left(T^{-1}(z)\right)=\alpha\left(q \circ T^{-1}\right)^{\prime}(z)=$ $p^{\prime}(z)$ and that $q^{\prime \prime}\left(T^{-1}(z)\right)=\alpha^{2} p^{\prime \prime}(z)$. Substituting these expressions in the formula for $H_{q}\left(T^{-1}(z)\right)$ we obtain

$$
\begin{aligned}
H_{q} \circ T^{-1}(z) & =\frac{z-\beta}{\alpha}-\frac{2 \alpha p(z) p^{\prime}(z)}{\alpha^{2}\left(2\left(p^{\prime}(z)\right)^{2}-p(z) p^{\prime \prime}(z)\right)} \\
& =\frac{z-\beta}{\alpha}-\frac{2 p(z) p^{\prime}(z)}{\alpha\left(2\left(p^{\prime}(z)\right)^{2}-p(z) p^{\prime \prime}(z)\right)} .
\end{aligned}
$$


Therefore

$$
\begin{aligned}
T \circ H_{q} \circ T^{-1}(z) & =T\left(\frac{z-\beta}{\alpha}-\frac{2 p(z) p^{\prime}(z)}{\alpha\left(2\left(p^{\prime}(z)\right)^{2}-p(z) p^{\prime \prime}(z)\right)}\right) \\
& =\alpha\left(\frac{z-\beta}{\alpha}-\frac{2 p(z) p^{\prime}(z)}{\alpha\left(2\left(p^{\prime}(z)\right)^{2}-p(z) p^{\prime \prime}(z)\right)}\right)+\beta \\
& =z-\frac{2 p(z) p^{\prime}(z)}{2\left(p^{\prime}(z)\right)^{2}-p(z) p^{\prime \prime}(z)} \\
& =H_{p}(z) ;
\end{aligned}
$$

that is, $T \circ H_{q} \circ T^{-1}=H_{p}$ and the proof is complete.

Next let $\tilde{q}(z)=b p(z)$ where $b$ is a constant. An easy computation then yields $H_{\tilde{q}}=H_{p}$; that is, the identity map is a conjugacy between the maps $H_{q}$ and $H_{\tilde{q}}$, thus their dynamics are equivalent.

Using the above results we may now prove the following result.

Theorem 1. Let $p(z)=(z-a)(z-b)(z-c)$ be a cubic polynomial with its roots ordered as follows: $0 \leq|a| \leq|b| \leq|c|$. Let $T(z)=(c-$ $a) z+a$. Then $q_{\lambda, \rho}(z)=p \circ T(z)=\lambda^{3} z(z-1)(z-\rho)$, where $\lambda=c-a$ and $\rho=\frac{b-a}{c-a}$, and $T$ is a conjugacy between $H_{p}$ and $H_{q_{\lambda, \rho}}$; that is, $T^{-1} \circ H_{q_{\lambda, \rho}} \circ T=H_{p}$. Furthermore if $\tilde{q}_{\rho}(z)=z(z-1)(z-\rho)$, then $H_{p}$ is conjugated with $H_{\tilde{q}_{\rho}}$.

Theorem 2. Let $p(z)=(z-a)(z-b)(z-c)(z-d)$ be a four degree generic polynomial. Assume that the roots of $p(z)$ are distinct and ordered as follows: $0 \leq|a| \leq|b| \leq|c| \leq|d|$. Let $T(z)=(d-a) z+a$, and let $q(z)=p \circ T(z)$. Then $T \circ H_{q_{\lambda, \rho, \mu}} \circ T^{-1}=H_{p}$ (that is, the Halley iteration functions of $q_{\lambda, \rho, \mu}(z)$ and of $p(z)$ are conjugated), where $q_{\lambda, \rho, \mu}(z)=p(T(z))=\lambda z(z-\rho)(z-\mu)(z-1), \lambda=(d-a)^{4}$, $\rho=\frac{b-a}{d-a}$, and $\mu=\frac{c-a}{d-a}$. Furthermore, $H_{q_{\lambda, \rho, \mu}}$ is conjugated to $H_{q_{\rho, \mu}}$ where $q_{\rho, \mu}(z)=z(z-\rho)(z-\mu)(z-1)$. 


\section{König Iteration Functions}

Now we study the König iteration functions.

Let $p(z)$ be a generic complex polynomial (that is, all of its roots distinct), and let $m$ be a positive integer greater or equal than two, the König iteration function $K_{m}(p)$ is an iteration function of order $m$ defined as follows

$$
K_{m, p}(z)=z+(m-1) \frac{\left(\frac{1}{p(z)}\right)^{(m-2)}}{\left(\frac{1}{p(z)}\right)^{(m-1)}} .
$$

For example, for $m=3$ and $m=4$, we have

$$
K_{3, p}(z)=z-\frac{2 p(z) p^{\prime}(z)}{2\left(p^{\prime}(z)\right)^{2}-p(z) p^{\prime \prime}(z)}
$$

and

$$
K_{4, p}(z)=z-\frac{3 p(z)\left(p(z) p^{\prime \prime}(z)-2\left(p^{\prime}(z)\right)^{2}\right)}{6 p(z) p^{\prime}(z) p^{\prime \prime}(z)-6\left(p^{\prime}(z)\right)^{3}-(p(z))^{2} p^{\prime \prime}(z)} .
$$

Note that $K_{2, p}=N_{p}$ and that $K_{3, p}=H_{p}$.

It is clear from equation (3) that the construction of $K_{m, p}$ requires the computation of the first $m-1$ derivative of $p(z)$. From [2], letting $h_{1}(z)=1$ and $h_{k+1}(z)=h_{k}^{\prime}(z) p(z)-k h_{k}(z) p^{\prime}(z)$, for $k=$ $1,2, \ldots, m-1$, we have

$$
\left(\frac{1}{p(z)}\right)^{(k)}=\frac{h_{k+1}(z)}{(p(z))^{k+1}}
$$

Thus equation (3) becomes

$$
K_{m, p}(z)=z+(m-1) \frac{h_{m-1}(z) p(z)}{h_{m}(z)} .
$$

Now if $q(z)=a p(z)$, where $a \neq 0$ is a constant, an easy computation shows that $K_{m, q}=K_{m, p}$.

Let $T(z)=\alpha z+\beta$, with $\alpha \neq 0$, be an affine map in $\mathbf{C}$. Let $q(z)=p \circ T(z)$. We have the following result. 
Theorem 1. (Scaling theorem König iteration functions) Let $p(z)$ be a polynomial. Let $T(z)=\alpha z+\beta$ with $\alpha \neq 0$ be an affine map, and let $q(z)=p \circ T(z)$. Then, for $m=2,3 \ldots$, we have $T$ is a conjugacy between $K_{m, q}$ and $K_{m, p}$.

Proof. We give the proof by induction, for $m=2$ we have $K_{2, p}=$ $N_{p}$. Now

$$
T \circ K_{q, m} \circ T^{-1}(z)=z+\alpha(m-1) \frac{h_{q, m}\left(T^{-1}(z)\right) q\left(T^{-1}(z)\right)}{h_{q, m}\left(T^{-1}(z)\right)}
$$

On the other hand, we have $h_{q, 1}\left(T^{-1}(z)\right)=h_{p, 1}(z)=1$. We claim that $h_{q, k}\left(T^{-1}(z)\right)=\alpha^{k-1} h_{p, k}(z)$. In fact, by induction, the assertion is true for $k=1$. Suppose $h_{q, k} \circ T^{-1}(z)=\alpha^{k-1} h_{p, k}(z)$ then $h_{q, k}^{\prime}\left(T^{-1}(z)\right)=$ $\alpha^{k} h_{p, k}^{\prime}(z)$. Thus,

$$
\begin{aligned}
h_{q, k+1}\left(T^{-1}(z)\right) & =h_{q, k}^{\prime}\left(T^{-1}(z)\right) q\left(T^{-1}(z)\right)-k h_{q, k}\left(T^{-1}(z)\right) q^{\prime}\left(T^{-1}(z)\right) \\
& =\alpha^{k} h_{p, k}(z) p(z)-k \alpha^{k-1} h_{p, k} \alpha p^{\prime}(z) \\
& =\alpha^{k}\left(h_{p, k}^{\prime}(z) p(z)-k h_{p, k}(z) p^{\prime}(z)\right) \\
& =\alpha^{k} h_{p, k+1}(z) .
\end{aligned}
$$

Replacing in $h_{q, k}\left(T^{-1}(z)\right)=\alpha^{k-1} h_{p, k}(z)$ in (5) we obtain

$$
\begin{aligned}
T \circ K_{q, m} \circ T^{-1}(z) & =z+\alpha(m-1) \frac{\alpha^{m-2} h_{p, m-1}(z) p(z)}{\alpha^{m-1} h_{p, m}(z)} \\
& =z+(m-1) \frac{h_{p, m-1}(z) p(z)}{h_{p, m}(z)} \\
& =K_{p, m}(z)
\end{aligned}
$$

and the proof is complete.

\section{Schröder Iteration Functions}

We now study the Schröder iteration function.

Let $p(z)$ be a generic complex polynomial (that is, all of its roots distinct), and let $m$ be a positive integer greater or equal than two. 
The Schröder iteration function $S_{m}(p)$ is an iteration function of order $m$.

We now show how to obtain the Schröder iteration function (for more details see [22]). If $|h|$ is small enough, then

$$
F(z+h)=F(z)+\sum_{n=1}^{\infty} b_{n}(z) h^{n}=F(z)+B(z)
$$

where $b_{n}(z)=\frac{F^{(n)}(z)}{n !}, n=1,2, \ldots$ The function $B(z)$ may be considered as a formal power series in the variable $h$ whose coefficients depend on $z$. If $b_{1}(z)=F^{\prime}(z) \neq 0$, then $B(z)$ may be inverted and

$$
B^{-1}(z)=\sum_{n=1}^{\infty} c_{n}(z) h^{n}
$$

where $c_{n}(z)=\frac{1}{n} \operatorname{res}\left(B^{-n}\right), n=1,2, \ldots$ (Lagrange-Bürmann formula see $[17])$.

Now using the above approximation of an analytic function, we may define the Schröder iteration function of order $m=2,3, \ldots$ for a polynomial $p(z)$ as follows:

$$
S_{m, p}(z)=z+\sum_{k=1}^{m-1} c_{k}(z)(-p(z))^{k} .
$$

It is easy see that

$$
c_{n}(z)=\frac{1}{k !}\left(\frac{1}{p^{\prime}(z)} \frac{d}{d z}\right)^{k-1} \frac{1}{p^{\prime}(z)}
$$

where

$$
\left(\frac{1}{p^{\prime}(z)} \frac{d}{d z}\right)^{k-1}=\underbrace{\left(\frac{1}{p^{\prime}(z)} \frac{d}{d z}\right)\left(\frac{1}{p^{\prime}(z)} \frac{d}{d z}\right) \cdots\left(\frac{1}{p^{\prime}(z)} \frac{d}{d z}\right)}_{(k-1)-\text { factors }}
$$

and that the coefficients $c_{n}(z)$ are analytic functions in every region $R \subset \mathbf{C}$ where $p^{\prime}(z) \neq 0$. 
For example, the order 3 and order 4 Schröder iteration functions of $p(z)$ are given by

$$
S_{3, p}(z)=z-\frac{p(z)}{p^{\prime}(z)}-\frac{p^{\prime \prime}(z)(p(z))^{2}}{2\left(p^{\prime}(z)\right)^{3}}
$$

and

$S_{4, p}(z)=z-\frac{p(z)}{p^{\prime}(z)}-\frac{p^{\prime \prime}(z)(g(z))^{2}}{2\left(p^{\prime}(z)\right)^{3}}-\frac{\left(\frac{1}{2}\left(p^{\prime \prime}(z)\right)^{2}-\frac{1}{6} p^{\prime}(z) p^{\prime \prime \prime}(z)\right)(p(z))^{3}}{\left(p^{\prime}(z)\right)^{5}}$.

Note that $S_{2, p}=N_{p}$; that is, the Schröder iteration function of order 2 corresponds to the Newton iteration function.

In order to obtain a formula for $S_{m, p}$, we follows [3] letting $h_{1}(z)=$ 1 and $h_{k+1}(z)=h_{k}^{\prime}(z) p^{\prime}(z)-(2 k-1) h_{k}(z) p^{\prime \prime}(z)$, for $k=1,2, \ldots$, we have

$$
\frac{1}{p^{\prime}(z)}\left(\frac{h_{k}(z)}{\left(p^{\prime}(z)\right)^{2 k-1}}\right)^{\prime}=\frac{h_{k+1}(z)}{\left(p^{\prime}(z)\right)^{2 k+1}}
$$

Thus equation (6) becomes

$$
S_{m, p}(z)=z+\sum_{k=1}^{m-1} \frac{(-1)^{k}}{k !} \frac{h_{p, k}(z)}{\left(p^{\prime}(z)\right)^{2 k-1}}(p(z))^{k}
$$

Now we have the following theorem.

Theorem 1. (Scaling theorem for Schröder iteration functions) Let $p(z)$ be a polynomial and let $T(z)=\alpha z+\beta$ de an affine map, and let $q(z)=p \circ T(z)$, then for $m=3,4, \cdots$, we have $T$ is a conjugacy between $S_{m, q}$ and $S_{m, p}$.

Proof. We have $S_{m, p}(z)=z+\sum_{k=1}^{m-1} \frac{(-1)^{k}}{k !} \frac{h_{p, k}(z)}{\left(p^{\prime}(z)\right)^{2 k-1}}(p(z))^{k}$. Thus

$$
\begin{aligned}
& T \circ S_{q, m} \circ T^{-1}(z) \\
& =z+\alpha \sum_{k=1}^{m-1} \frac{(-1)^{k}}{k !} \frac{h_{q, k}\left(T^{-1}(z)\right)}{\left(q^{\prime}\left(T^{-1}(z)\right)\right)^{2 k-1}}\left(q\left(T^{-1}(z)\right)\right)^{k} .
\end{aligned}
$$


Since, $q \circ T^{-1}(z)=p(z)$ we have $q^{\prime}\left(T^{-1}(z)\right)=\alpha p^{\prime}(z)$ and the above expression becomes

$$
T \circ S_{q, m} \circ T^{-1}(z)=z+\sum_{k=1}^{m-1} \frac{(-1)^{k}}{k !} \frac{h_{q, k}\left(T^{1}(z)\right)}{\alpha^{2 k-2}\left(p^{\prime}(z)\right)^{2 k-1}}(p(z))^{k}
$$

Now, we have $h_{q, 1}\left(T^{-1}(z)\right)=1=h_{p, 1}(z)$. We claim that for $k=$ $1,2, \ldots$

$$
h_{q, k}\left(T^{-1}(z)\right)=\alpha^{2 k-2} h_{p, k}(z) .
$$

We prove (9) by induction. It is clear for $k=1$, and for $k=2$ and $k=3$ the verification is a straightforward computation. Suppose that $h_{q, k} \circ T^{-1}(z)=\alpha^{2 k-2} h_{p, k}(z)$. We have $h_{q, k}\left(T^{-1}(z)\right)=\alpha^{2 k-1} h_{p, k}(z)$ and obtain

$$
\begin{aligned}
h_{q, k+1}\left(T^{-1}(z)\right) & =\alpha^{2 k}\left(h_{p, k}^{\prime}(z) p^{\prime}(z)-(2 k-1) h_{p, k}(z) p^{\prime \prime}(z)\right) \\
& =\alpha^{2 k} h_{p, k}(z)
\end{aligned}
$$

Replacing, $h_{q, k}\left(T^{-1}(z)\right)=\alpha^{2 k-k} h_{p, k}(z)$ in (9) we obtain

$T \circ S_{m, q} \circ T^{-1}(z)=z+\sum_{k=1}^{m-1} \frac{(-1)^{k}}{k !} \frac{\alpha^{2 k-2} h_{p, k}(z)}{\alpha^{2 k-2}\left(p^{\prime}(z)\right)^{2 k-1}}(p(z))^{k}=S_{p, m}(z)$,

and the proof is complete.

We now have the next two results. Their proofs follow from Theorems 5 and 6 , a straightforward computation.

Theorem 2. Let $p(z)=(z-a)(z-b)(z-c)$ be a cubic polynomial with its roots distinct and ordered as follows: $0 \leq|a| \leq|b| \leq|c|$. Let $T(z)=(c-a) z+a$, and let $q(z)=p \circ T(z)$. Then $q_{\lambda, \rho}(z)=$ $\lambda^{3} z(z-1)(z-\rho)$ where $\lambda=c-a$ and $\rho=\frac{b-a}{c-a}$. For $m=2,3,4, \cdots$, we have that $T$ is a conjugacy between $S_{m, q_{\lambda, \rho}}$ and $S_{m, p}$ as well as between $K_{m, q_{\lambda, \rho}}$ and $K_{m, p}$. Furthermore, if $\tilde{q}(z)=z(z-1)(z-\rho)$, for $m=2,3,4, \cdots$, we have that $S_{m, \tilde{q}_{\rho}}$ and $S_{m, q_{\lambda, \rho}}$ are conjugated, and so are $K_{m, \tilde{q}_{\rho}}$ and $K_{m, q_{\lambda, \rho}}$.

Therefore $T$ is a conjugacy between $S_{m, \tilde{q}}$ and $S_{m, p}$ as well as between $K_{m, \tilde{q}}$ and $K_{m, p}$, for $m=2,3,4, \cdots$. 
Theorem 3. Let $p(z)=(z-a)(z-b)(z-c)(z-d)$ be a four degree polynomial with its roots distinct and ordered as follows: $0 \leq|a| \leq$ $|b| \leq|c| \leq|d|$. Let $T(z)=(d-a) z+a$, and let $q(z)=p \circ T(z)$. Then $q(z)=\lambda^{4} z(z-1)(z-\rho)(z-\mu)$ where $\lambda=d-a, \quad \rho=\frac{b-a}{d-a}$, and $\mu=\frac{c-a}{d-a}$. For $m=3,4$, we have that $T$ is a conjugacy between $S_{m, q}$ and $S_{m, p}$ as well as between $K_{m, q}$ and $K_{m, p}$. Furthermore if $\tilde{q}(z)=z(z-1)(z-\rho)(z-\mu)$, for $m=3,4$, we have that $S_{m, \tilde{q}}$ and $S_{m, q}$ are conjugated, and so are $K_{m, \tilde{q}}$ and $K_{m, q}$.

In [12], the respective space of parameter of the Schröder iteration functions $S_{3}(z)$ and $S_{4}(z)$ associated to the family of cubic polynomials $q_{A}(z)=z^{3}+(A-1) z-A$ is studied. Since $T \circ S_{m, \tilde{q}_{A, \mu}} \circ T^{-1}=S_{\tilde{q}_{\rho}}$, for $m=3,4$, (which follows from reducing the family of generic cubic polynomials $\tilde{q}_{\rho}(z)=z(z-\rho)(z-1)$ to the family $\tilde{q}_{A, \mu}(z)=\mu\left(z^{2}+z+\right.$ $A)(z-1)=\mu\left(z^{3}+(A-1) z-A\right)$ using the map $T(z)=\frac{2-\rho}{3} z+\frac{1+\rho}{3}$, that is, $\left.\tilde{q}_{A, \mu}(z)=q_{\rho} \circ T(z)\right)$, and since $S_{m, \tilde{q}_{A}}=S_{m, \tilde{q}_{A, \mu}}$, it suffices to study the dynamics of $S_{m, \tilde{q}_{A}}$.

On the other hand, note that

$$
\begin{aligned}
q_{\rho, \mu}(z) & =z(z-\rho)(z-\mu)(z-1) \\
& =\left(z^{2}-\rho z\right)(z-\mu)(z-1) \\
& =\left(z^{2}-\rho z\right)\left(z^{2}-z \mu z+\mu\right) \\
& =\left(z^{2}-\rho z\right)\left(z^{2}-\left(z^{2}-(\mu+1) z+\mu\right)\right) \\
& =z^{4}-(\mu+1) z^{3}+\mu z^{2}-\rho z^{3}+\rho(\mu+1) z^{2}-\rho \mu z \\
& =z^{4}-(\rho+\mu+1) z^{3}-(\mu+\rho(\mu+1)) z^{2}-\rho \mu z
\end{aligned}
$$

hence $q_{\rho, \mu}(z)=z^{4}-(\rho+\mu+1) z^{3}+(\mu+\rho(\mu+1)) z^{2}-\rho \mu z$. This is a two-parameter family of polynomials, a study of the $(\rho, \mu)$-space may be done. For example, the parameter space of a special family of a four degree generic polynomial $q_{\alpha}(z)=z^{4}+(\alpha-1) z^{2}-\alpha$, which has roots $z_{1}=1, z_{2}=-1, z_{3}=\sqrt{\alpha} i$ and $z_{4}=-\sqrt{\alpha} i$, was studied in $[4]$. 


\section{References}

[1] Alexander, D. S. A history of complex dynamics: from Schröder to Fatou and Julia. Vieweg, Aspects of Mathematics (1994).

[2] Argiropoulos, N., Drakopoulos, V., Böhm, A., Julia and Mandelbrot-like sets for higher order König rational iteration functions. Fractal Frontier, M. M. Novak and T. G. Dewey, eds. World Scientific, Singapore, 169-178, (1997).

[3] Argiropoulos, N., Drakopoulos, V., Böhm, A., Generalized computation of Schröder iteration functions to motivate families of Julia and Mandelbrot-like sets. SIAM J. Numer. Anal., Vol. 36, No 2, pp. 417-435, (1999).

[4] Arney, D. C., Robinson, B. T. Exhibiting chaos and fractals with a microcomputer. Comput. Math. Applic. Vol. 19 (3), pp. 1-11, (1990).

[5] Ben-Israel, A. Newton's method with modified functions. Contemporary Mathematics 204, pp. 39-50, (1997).

[6] Ben-Israel A., Yau, L. The Newton and Halley method for complex roots. The American Mathematical Monthly 105, pp. 806818, (1998).

[7] Blanchard, P. Complex Analytic Dynamics on the Riemann sphere. Bull. of AMS (new series) Vol. 11, number 1, July, pp. 85-141, (1984).

[8] Blanchard, P., Chiu, A. Complex Dynamics: an informal discussion. Fractal Geometry and Analysis. Eds. J. Bélair \& S. Dubuc. Kluwer Academic Publishers, pp. 45-98, (1991).

[9] Cayley, A. The Newton-Fourier Imaginary Problem. Amer. J. Math. 2, 97, (1879). 
[10] Cayley, A. On the Newton-Fourier Imaginary Problem. Proc. Cambridge Phil. Soc. 3, pp. 231-232, (1880).

[11] Curry, J. H., Garnett, L., Sullivan, D. On the iteration of a rational function: computer experiment with Newton method. Comm. Math. Phys. 91, pp. 267-277, (1983).

[12] Drakopoulos, V. On the additional fixed points of Schröder iteration function associated with a one-parameter family of cubic polynomilas. Comput. and Graphics, Vol. 22 (5), pp. 629-634, (1998).

[13] Douady A., Hubbard, J. H. On the dynamics of polynomial-like mappings. Ann. Sci. Ec. Norm. Sup. (Paris) 18 (1985), 287-343.

[14] Gilbert, W. Newton's method for multiple roots. Comput. and Graphics, Vol. 18 (2), pp. 227-229, (1994).

[15] Gilbert, W. The complex dynamics of Newton's method for a double root. Computers Math. Applic., Vol. 22 (10), pp. 115-119, (1991).

[16] Emerenko, A., Lyubich, M., Y. The Dynamics of Analytic Transformations. Leningrad Math. J., Vol. 1 (3), pp. 563-634, (1990).

[17] Henrici, P. Applied and Computational Compex Analysis. Wiley, (1974).

[18] Milnor, J. Dynamics in One Complex Dimension: Introductory Lectures. Preprint \#1990/5, SUNY StonyBrook, Institute for Mathematical Sciences.

[19] Peitgen, Heinz - Otto, (Ed.) Newton's Method and Dynamical Systems. Kluwer Academic Publishers, (1989).

[20] Schröder, E. O. On infinitely many algorithms for solving equations. Math. Ann. 2 (1870), pp. 317-265. Translated by G. W. Stewart, 1992 (these report is available by anonymous ftp from thales.cs.umd.edu in the directory pub/reports). 
[21] Vrscay, E. R. Julia sets and Mandelbrot-like sets associated with higher order Schröder rational iteration functions: a computer assisted study. Mathematics of Computation, Vol. 46 (173), pp. 151-169, (1986).

[22] Vrscay, E. R., Gilbert W. J. Extraneous fixed points, basin boundary and chaotic dynamics for Schröder and König rational iteration functions. Numer. Math. 52, pp. 1-16, (1988).

Received : February 2001.

\section{Sergio Plaza Salinas}

Departamento de Matemáticas y Cs. de la Computación Universidad de Santiago de Chile

Casilla 307

Correo 2

Santiago

Chile

e-mail : splaza@fermat.usach.cl 